\title{
Laparoscopic Excision of Functional Ganglioneuroma Near Renal Hilum
}

\author{
PRK Bhargav, V Amar, SK Uday
}

\begin{abstract}
Ganglioneuromas are one of the frequent retroperitoneal tumors arising from extraadrenal sympathetic ganglia or chromaffin tissue. The commonest extraadrenal site is periadrenal region near renal hilum. We present a case of ganglioneuroma in a narrow quadrangular space near right renal hilum, treated laparoscopically.
\end{abstract}

Keywords: Ganglioneuroma, Renal hilum, Laparoscopic excision.

How to cite this article: Bhargav PRK, Amar V, Uday SK. Laparoscopic Excision of Functional Ganglioneuroma Near Renal Hilum. World J Endocr Surg 2012;4(2):63-65.

\section{Source of support: Nil}

Conflict of interest: None declared

\section{INTRODUCTION}

Paragangliomas and ganglioneuromas can arise from extraadrenal sympathetic ganglia or chromaffin tissue of retroperitoneum. ${ }^{1}$ Surgery either with open or laparoscopic approach is the treatment of choice for these tumors. We describe a case of functional ganglioneuroma arising from retroperitoneum in a precarious areas near right renal hilum managed by successful laparoscopic excision.

\section{CASE REPORT}

A 28-year-old lady presented with nonradiating pain in right loin since 3 months. There was no history of gastrointestinal disturbance or urinary complaints. She was detected to have episodic hypertension with maximum blood pressure of 170/110 mm Hg. There was no history of classic triad of headache, sweating and palpitations, which is suggestive of pheochromocytoma. There was no family history or stigmata of multiple endocrine neoplasia or familial paraganglioma syndromes. Computerized axial tomogram (CT scan) showed a $4 \times 4 \mathrm{~cm}$ heteroechoic mass in the right adrenal region close to the renal hilar vasculature (Fig. 1). The patient and first-degree relatives were clinically screened for syndromic associations, such as MEN syndrome, neurofibromatosis, paraganglioma syndrome, Von-Hippel Lindau disease, Struge-Weber syndrome and tuberous sclerosis, but no suggestive stigmata was found. Genetic testing of MEN 2 syndrome was normal. There was no thyromegaly or thyroid nodularity on sonography. According to our departmental protocol, other causes of secondary hypertension, such as renal, vascular, neurological, drug abuse were excluded by clinical evaluation and relevant investigations. Twenty-four hours urinary total metanephrine level was $1.2 \mathrm{mg}(0.7-0.95)$, plasma metanephrine level was $29 \mathrm{pg} / \mathrm{ml}(<80)$ and 24 hours urinary vanillylmandelic acid level (VMA) was $11.4 \mathrm{mg}(<8 \mathrm{mg})$. Serum calcium, phosphorus and alkaline phosphatase were within normal limits. The latter was particularly suggestive of a functional chromaffin tumor. In view of secondary hypertension in a young lady with evidence of tumor in right adrenal region a diagnosis of pheochromocytoma or neurochromaffin tumor was considered, though metanephrine levels were marginally elevated. Preoperative preparation was done with titrated escalating doses of prazosin (maximum $=8 \mathrm{mg} / \mathrm{d}$ ), fluid and high salt intake.

Operative details: After 9 days of preparation, she was taken up for surgery. Approach was transperitoneal laparoscopic with four ports in right hypochondrium and lumbar regions. Contrary to our first diagnosis of adrenal originated pheochromocytoma, we found a highly vascular tumor being unrelated to the adrenal gland. It was located in the retroperitoneum in a narrow quadrangular space flanked by renal hilum, ureter on right, renal vessels superiorly, inferior vena cava on left side and right colic flexure inferiorly (Fig. 2). Schematic diagram of the same laparoscopic view is depicted in Figure 3. With gentle dissection using bipolar diathermy and hydrodissection with irrigation/suction

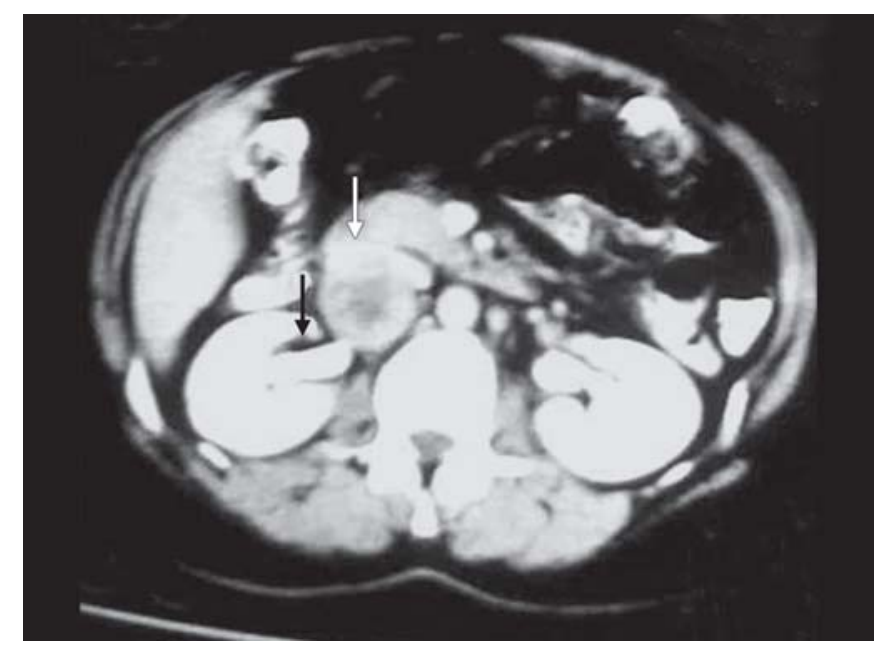

Fig. 1: Computerized tomogram of abdomen-heterogeneous mass (white arrow) in adrenal area abutting renal vasculature (black arrow) 


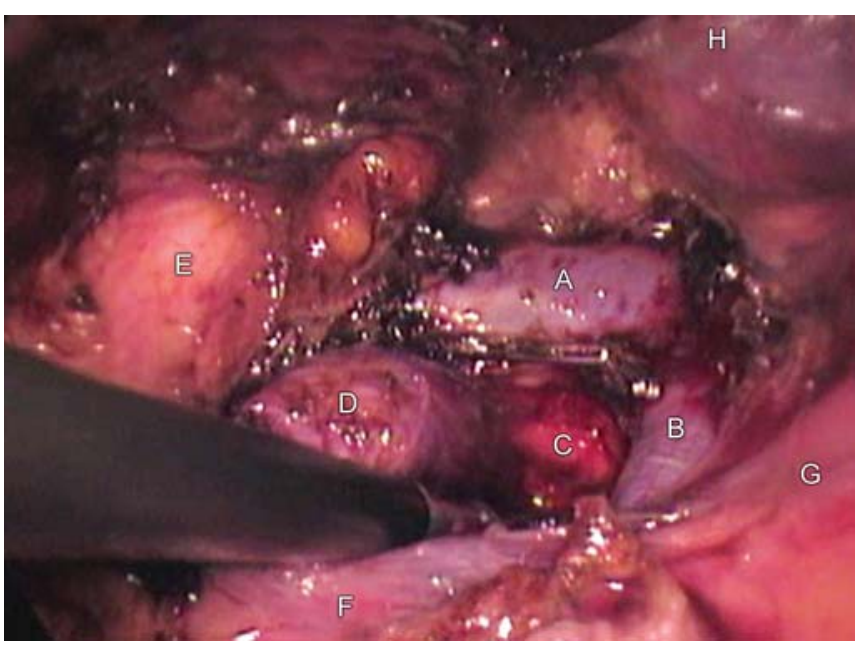

Fig. 2: Intraoperative view of tumor: (A) right renal vein; (B) inferior vena cava; $(C)$ renal artery; $(D)$ tumor; $(E)$ right kidney; $(F)$ hepatic colic flexure; $(\mathrm{G})$ duodenum; $(\mathrm{H})$ liver

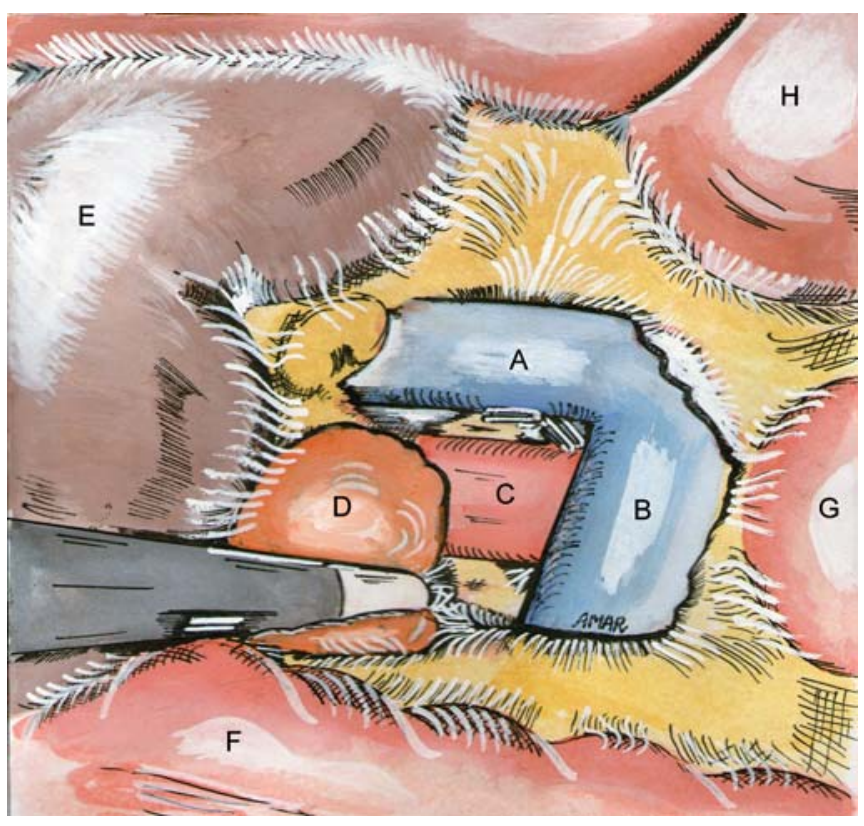

Fig. 3: Schematic diagram of structures shown in Figure 2

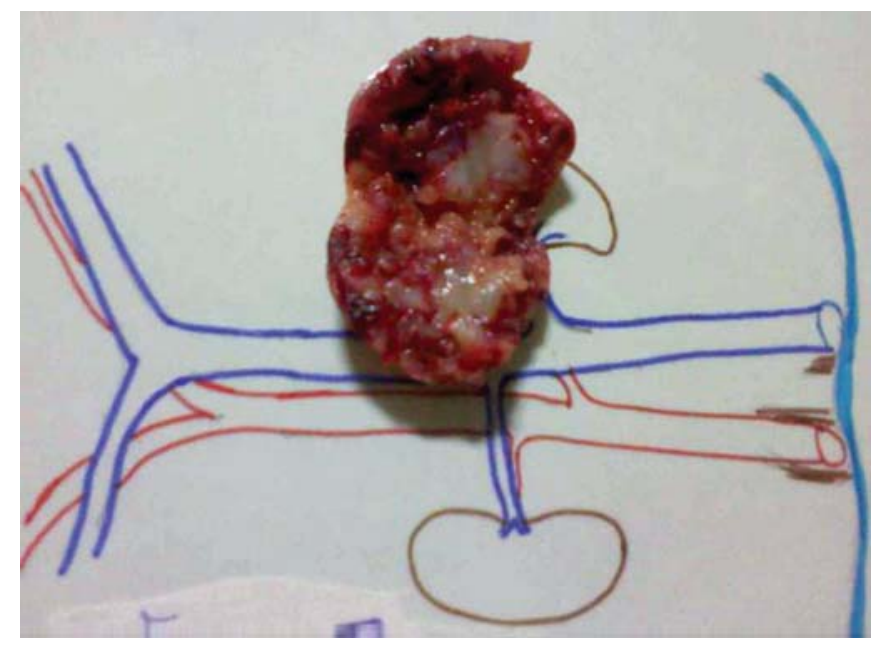

Fig. 4: Cut section of ex vivo specimen of tumor on grossing chart cannula, intact tumor without any capsular breach was extirpated without injury to adjacent vital structures. The extirpated ex vivo specimen is shown in Figure 4. Postoperative period was uneventful with mild hypertension of 136/90 mm Hg and patient was put on angiotensin inhibitor. Histopathology of the tumor was suggestive of a benign ganglioneuroma with schwannian stroma consisting of Antoni A areas intermixed with mature, few immature ganglion cells and chromaffin cells (Fig. 5). Immunohistochemistry demonstrated reactivity for neuron-specific enolase and chromogranin A. On regular follow-up, she became normotensive at 5 months follow-up.

\section{DISCUSSION}

Neurochromaffin tumors, such as paragangliomas and ganglioneuromas usually arise from lumbar or thoracic sympathetic ganglia and rarely in the neck and skull base. ${ }^{2}$ Occasionally, mixed ganglioneuromapheochromocytoma may occur suggesting their common origin from neural crest of embryonic neurectoderm. ${ }^{2}$ Ganglioneuromas can be associated with disturbance in catecholamine metabolism and secretion of dopamine, VMA in contrast to metanephrine or normetanephrine as in pheochromocytoma. ${ }^{3}$ Serum chromogranin A is a nonspecific marker for neuroendocrine tumors, which is especially useful in a unknown tumor pathology to predict its nature in the setting of normal levels of specific markers (in this case metanephrines/VMA). We evaluate chromogranin A level in a case of neuroendocrine tumor of unknown origin in the absence of elevated specific markers or in the follow-up monitoring after treatment of neuroendocrine tumor. As seen in this case, the tumor had a predominantly neuronal differentiation with few chromaffin elements as confirmed by histopathology and immunohistochemistry.

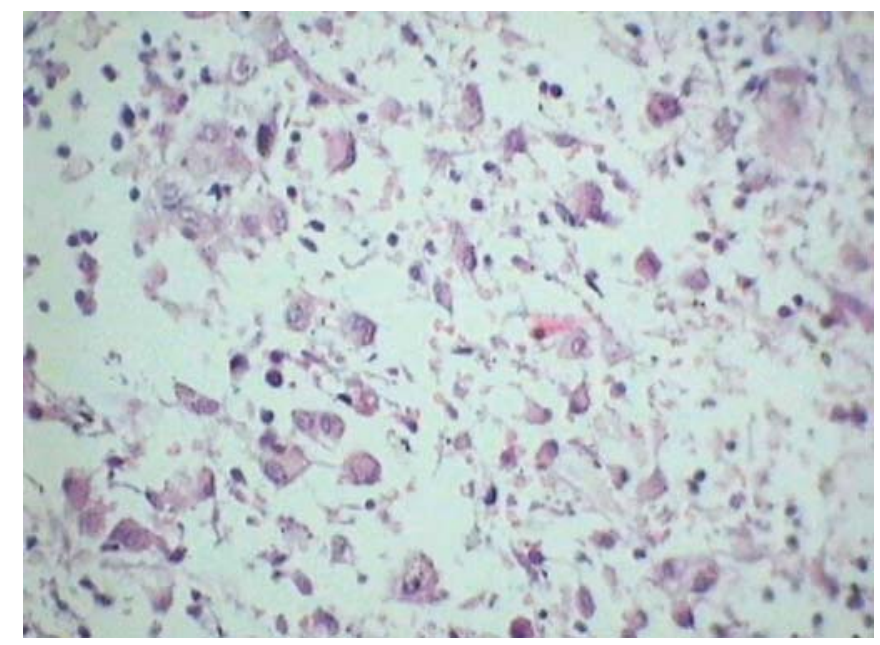

Fig. 5: Microphotograph of the tumor showing combination of ganglion cells (predominantly) and chromaffin elements interspersed in a scanty neuronal stroma 
The commonest location of EAP and extraadrenal ganglioneuroma (EAGN) is periadrenal area and renal hilum is one of the most frequent site of these tumors. ${ }^{1}$ Sometimes, the differentiation between adrenal and extraadrenal masses in the vicinity is a difficult task. Though, the present thin slice computed tomography (CT) scan with reconstructional protocols offer a virtual intra-abdominal topography of the retroperitoneal tumors, especially in three-dimensional and coronal sections. ${ }^{4}$ But, we have not performed any CT scan reconstruction of the tumor, which has resulted in our broader differential diagnosis. The role of preoperative MIBG scintigraphy is a debatable issue with both supportive and opposing data. In our department, we believe in postoperative MIBG scan and when planning MIBG therapy, because of its poor sensitivity ${ }^{5,6}$ for smaller tumor sites, metastatic deposits and familial paragangliomas in the preoperative setting, which are masked by the primary tumor mass. Moreover, latest thin slice CT protocols serve the purpose better than preoperative MIBG scan. Their clinical presentation ranges from asymptomatic incidentaloma to abdominal lump or functional tumors. Functional neurogenic or chromaffin tumors are associated with hypertension secondary to hormonal hypersecretion or renal vascular compression. Surgery is usually done via open approach, though technical advances and surgical expertise allows laparoscopic approach. The laparoscopic excision of retroperitoneal ganglioneuromas can be arduous task due to precarious proximity to great vessels, often with adhesions, direct vascular supply or encasement of blood vessels. ${ }^{7-9}$ There have been few case reports and short series of laparoscopic excision of retroperitoneal tumors and EAGN, establishing their laparoscopic feasibility. ${ }^{8,10}$ There is a case report laparoscopic excision of ganglioneuroma using vessel seal device to separate it from posterior inferior vena cava. ${ }^{7}$ This case was done with successful use of electrocautery, hydrodissection without the use of harmonic shears, in spite of proximity of vital structures and direct vascular supply. With conventional CT scan, caution needs to be excised in differentiating between renal and extrarenal masses in the perirenal region, though reconstructional CT scan with coronal sections can serve this purpose better. ${ }^{4}$ This case further highlights the feasibility of laparoscopic excision of EAP, even in the precarious area of renal hilum in experienced hands.

\section{REFERENCES}

1. Whalen RK, Althausen AF, Daniels GH. Extra-adrenal pheochromocytoma. J Urol 1992;147:1-10.

2. Royer N, Miron J, Kirby P, Weigel RJ, Lal G. Incidental finding of composite pheochromocytoma-ganglioneuroma: Successful management after emergent appendectomy and review of the literature. World J Endocrine Surgery 2011;3:39-44.

3. Crout JR. Catecholamine metabolism in pheochromocytoma and essential hypertension. In: Manger WM, (Ed). Hormones and Hypertension. Springfield, IL: Charles C Thomas 1966;31.

4. Wu YH, Song B, Xu J, Chen WX, Zhao XF, et al. Retroperitoneal neoplasms within the perirenal space in infants and children: Differentiation of renal and non-renal origin in enhanced CT images. Eur J Radiol 2010;75:279-86.

5. Lumachi F, Tregnaghi A, Zucchetta P, et al. Sensitivity and positive predictive value of CT, MRI and 123I-MIBG scintigraphy in localizing pheochromocytomas: A prospective study. Nucl Med Commun 2006;27:583-87.

6. Bhatia KS, Ismail MM, Sachdev A, et al. 123I-metaiodobenzylguanidine (MIBG) scintigraphy for the detection of adrenal and extra-adrenal pheochromoctyomas: CT and MRI correlation. Clin Endocrinol (Oxf) 2008;69:181-88.

7. Oue T, Yoneda A, Sasaki T, Tani G, Fukuzawa M. Total laparoscopic excision of retroperitoneal ganglioneuroma using the hanging method and a vessel-sealing device. J Laparoendosc Adv Surg Tech A. 2008;18:779-82.

8. Ahn KS, Han HS, Yoon YS, Kim HH, et al. Laparoscopic resection of nonadrenal retroperitoneal tumors. Arch Surg 2011;146:162-67.

9. Nelms JK, Diner EK, Lack EE, Patel SV, Ghasemian SR, Verghese M. Retroperitoneal ganglioneuroma encasing the celiac and superior mesenteric arteries. Scientific World Journal 2004;4:974-77.

10. Sasaki A, Suto T, Nitta H, et al. Laparoscopic excision of retroperitoneal tumors: Report of three cases. Surg Today 2010;40:176-80.

\section{ABOUT THE AUTHORS}

\section{PRK Bhargav (Corresponding Author)}

Associate Professor, Department of Endocrine Surgery, Mamata Medical College and Superspeciality Hospital, Khammam, Andhra Pradesh, India, Phone: 91-9490130798, Fax: 08742-230862, 255718 e-mail: kingbhargav@gmail.com

\section{Amar}

Consultant Advanced Laparoscopic Surgeon, Prime Hospitals Hyderabad, Andhra Pradesh, India

\section{SK Uday}

Associate Professor, Department of General Surgery, Mamata Medical College and Superspeciality Hospital, Khammam, Andhra Pradesh, India 